\title{
Detection of Incomplete Left Bundle Branch Block by Non-invasive Electrocardiographic Imaging
}

\author{
Laura Bear $^{1,2,3}$, Peter Huntjens ${ }^{1,2,3,4}$, Ruben Coronel ${ }^{1,2,3,5}$, Olivier Bernus ${ }^{1,2,3}$, Corentin Dallet ${ }^{1,2,3}$, \\ Richard Walton ${ }^{1,2,3}$, Rémi Dubois ${ }^{1,2,3}$ \\ ${ }^{1}$ Electrophysiology and Heart Modelling Institute (IHU LIRYC), Bordeaux, France. \\ ${ }^{2}$ CRCTB Inserm U1045, Bordeaux, France \\ ${ }^{3}$ Université de Bordeaux, CRCTB Inserm U1045, Bordeaux, France \\ ${ }^{4}$ Dept. Biomedical Engineering, Maastricht University, Maastricht, The Netherlands \\ ${ }^{5}$ Dept. Exp. Cardiology, Academic Medical Center, Amsterdam, The Netherlands
}

\begin{abstract}
This study sought to compare noninvasive ECGi with local epicardial activation time mapping to detect subtle electrical dyssynchrony between the left and right ventricles. We used an ex-vivo porcine model of complete and incomplete left bundle branch block (LBBB) in an experimental torso-tank. LBBB was induced in Langendorff-perfused pig hearts suspended in tank shaped as a male torso fitted with 256 body surface electrodes and 108 epicardial electrodes. Epicardial and surface potentials were recorded during sinus rhythm and ventricular pacing. Post-experiment MRI provided the epicardial geometry and electrode (epicardial and surface) locations. Epicardial electrograms were calculated and the derived reconstructed values for electrical dyssynchrony were compared to those recorded.

$\angle B B B$ with varying levels of electrical delay was successfully induced in 7 out of 8 hearts, with incomplete $\angle B B B$ present in 4. ECGi correctly identified the presence/absence of dyssynchronous activity in 7 of the 8 cases, including incomplete $L B B B$ with very subtle delay $(V E U=17 \mathrm{~ms}, T A T=45 \mathrm{~ms})$. Overall, there was no significant difference between measured and calculated $\operatorname{VEU}(p=0.06)$, or TAT values $(p=0.21)$.
\end{abstract}

\section{Introduction}

In patients with systolic dysfunction combined with conduction abnormalities, delayed electrical activation of the left ventricle (LV) with respect to the right ventricle $(\mathrm{RV})$ is considered as the underlying cause of reduced $\mathrm{LV}$ function [1]. Non-invasive electrocardiographic imaging (ECGi) has been shown to be a more reliable technique than the 12-lead ECG to identify delayed LV activation in patient with heart failure, as it allows activation time calculation at thousands of epicardial sites [2]. It has been assumed that ECGi is able to accurately detect subtle electrical dyssynchrony, even in hearts without complete LBBB. However, validation of the ability of ECGi to detect delayed LV electrical activation has not yet been performed. In addition it is unknown how accurately ECGi can detect electrical dyssynchronous activation in a heart with relatively small conduction abnormalities such as incomplete LBBB.

In patients with LBBB and heart failure, biventricular (BiV) pacing can restore electrical synchrony [3]. The site of pacing is a major determinant of the benefit of this technique, with the optimal location and timing of $\mathrm{LV}$ pacing differing substantially between patients [4]. Despite this, the techniques to optimize the position of these leads clinically are limited.

This study presents an initial evaluation, aiming to: (1) validate the detection of electrical dyssynchrony markers by ECGi; (2) evaluate the detection of incomplete LBBB by ECGi and (3) evaluate the use of $\mathrm{BiV}$ pacing to restore electrical synchrony.

\section{Methods}

\subsection{Ex-vivo Experimental Data}

$E x$-vivo experimental data came from Langendorffperfused pig hearts perfused with a standard Tyrode's solution. A flexible electrode sock (18 electrodes) was placed over the epicardium. Control recordings were taken in sinus rhythm prior to LBBB induction. Pacing leads were placed on the RV apex and the LV freewall (slightly posterior). In each heart, LBBB was induced by one of two methods:

Method 1 ( $n=3)$ : Ligation of part of the left bundle branch. For LBBB induction the suture was pulled through, severing the branch. 
Method 2 ( $n=5)$ : Local radiofrequency ablation (25$30 \mathrm{~W}$ and a duration of 30-60 sec.).

Recordings were taken outside the tank for one complete LBBB heart. The other seven hearts were suspended inside a human-shaped tank, filled with oxygenated electrolytic solution of $37^{\circ} \mathrm{C}$. Of these hearts, LBBB induction was performed inside the tank for these cases. Tank potentials were recorded from 128 electrodes, simultaneously with epicardial potentials, pre- and postLBBB induction, during spontaneous rhythm, LV, RV and bi-ventricular $(\mathrm{BiV})$ pacing protocols. In the results, $\mathrm{m}$ refers to the number of hearts, of the eight, in which recordings were successfully obtained. Post-experiment MRI provided the epicardial geometry and electrode (epicardial and tank) locations.

\subsection{ECGi evaluation}

Electrograms were reconstructed onto experimentspecific ventricular epicardial geometries (up to 4000 points) using the method of fundamental solutions, an ECGi method often used clinically [5]. Activation times (ATs) were derived from the recorded and ECGi reconstructed potentials as the time of maximum negative derivative. A number of markers have been developed in conjunction with ECGi to describe quantitatively the electrical dyssynchrony within the heart [6,7]. In this study, the following have been used:

Ventricular electrical uncoupling (VEU): the difference in mean activation of the LV and RV free wall. A positive value reflects LV delay (relative to right ventricular wall), whereas a negative value reflects $\mathrm{RV}$ delay (relative to the left ventricular wall).

Total activation time (TAT): the total duration of activation over the epicardium.

RVTAT and LVTAT: the total duration of activation over each ventricular epicardium.

QRS-interval (QRSi): The width of the QRS defined manually with calipers from the 12-lead ECG. As tank potentials were not always available, this metric was estimated from epicardial potentials by computing a root-mean-square, then defining the width of the depolarization curve.

LBBB was defined when AT maps showed delayed electrical activation of the LV (positive VEU), with an increase in LVTAT and TAT. Complete LBBB was defined as TAT $\geq 65 \mathrm{~ms}$ and $\mathrm{VEU} \geq 40 \mathrm{~ms}$, while incomplete LBBB as $65 \mathrm{~ms}>\mathrm{TAT} \geq 35 \mathrm{~ms}$, and $40 \mathrm{~ms}>$ VEU $\geq 10 \mathrm{~ms}$. These values are consistent with the porcine model of LBBB developed in [8]. To determine differences between recorded and ECGi markers, a paired t-test was used. Statistical significance was accepted for $\mathrm{p}<0.05$.

\section{Results}

\subsection{Model of LBBB}

Table 1 displays a summary of the results across 8 hearts. Overall, control signals were taken from 4 of the hearts demonstrating no signs of delay in either the RV or LV. The other four hearts started with some degree of LBBB. This was due to cutting the LBB during ligation in two hearts and manipulations during placement of the ablation catheter in the other two.

Table 1. Summary of experimental recordings during normal sinus rhythm, pre- and post LBBB induction.

\begin{tabular}{lccc}
\hline & $\begin{array}{c}\text { Control } \\
(\mathbf{m}=\mathbf{4})\end{array}$ & $\begin{array}{c}\text { Incomplete } \\
\text { LBBB } \\
(\mathbf{m}=\mathbf{4})\end{array}$ & $\begin{array}{c}\text { Complete } \\
\text { LBBB } \\
(\mathbf{m}=3)\end{array}$ \\
\hline QRSi (ms) & $44 \pm 9$ & $64 \pm 16$ & $78 \pm 10^{*}$ \\
TAT (ms) & $25 \pm 3$ & $49 \pm 8^{*}$ & $72 \pm 7^{*}$ \\
LVTAT (ms) & $19 \pm 5$ & $31 \pm 12$ & $46 \pm 11^{*}$ \\
RVTAT (ms) & $12 \pm 3$ & $17 \pm 6^{*}$ & $18 \pm 2^{*}$ \\
VEU (ms) & $2 \pm 6$ & $26 \pm 10$ & $46 \pm 6^{*}+$ \\
\hline
\end{tabular}

${ }^{*} \mathrm{p}<0.05$ versus control. $\dagger \mathrm{p}<0.05$ versus incomplete LBBB.

Overall, LBBB induction was successfully developed in 7 hearts. One of the ablated hearts, showed no signs of LBBB. That is, all markers lay within the control range, and analysis of the endocardium after the experiments completion revealed burn marks missing the bundle branch. Fig 1 presents examples of activation maps pre and post LBBB induction. Top panels represent the electrode sock wrapped around the epicardial surface (red), and bottom panels represent unfolded activation maps (see diagram). Complete LBBB was present in 3 hearts, and incomplete in 4 hearts. Despite these definitions, there was a wide spread in the degree of delay seen in these hearts, with VEU ranging from $15 \mathrm{~ms}$ to $52 \mathrm{~ms}$, and TAT from 42 $\mathrm{ms}$ to $79 \mathrm{~ms}$. The LV AT delay produced with ablation was typically longer than with ligation (TAT $=54 \pm 15 \mathrm{~ms}$ vs $72 \pm 6 \mathrm{~ms}$ and $\mathrm{VEU}=43 \pm 6 \mathrm{~ms}$ vs $27 \pm 13 \mathrm{~ms}$ ).

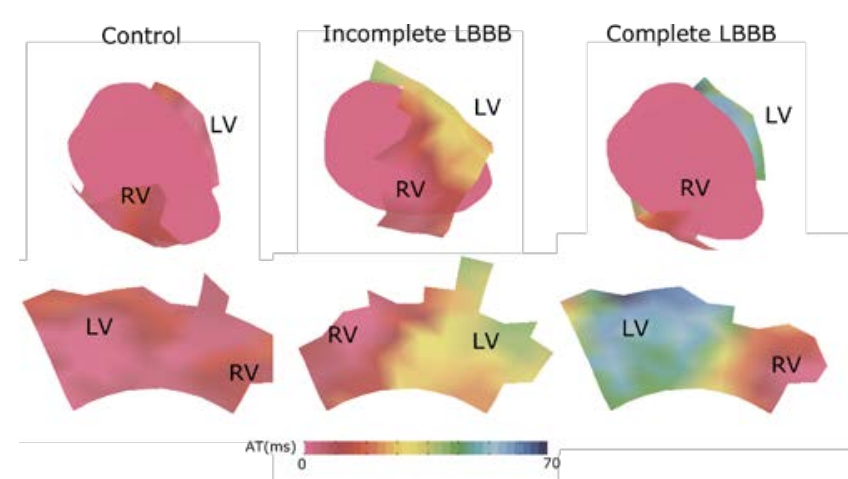

Fig 1. Examples of control, incomplete and complete LBBB activation maps. 


\subsection{ECGi evaluation}

Fig 2 presents examples of recorded and ECGi derived activation maps in (a) complete and (b) incomplete LBBB. Recorded maps are shown on sock geometries overlaying the epicardial surface. Both ECGi maps capture late activation over the LV free wall, and nearer to the base in (b), however the TAT duration is reduced slightly in both relative to recorded.
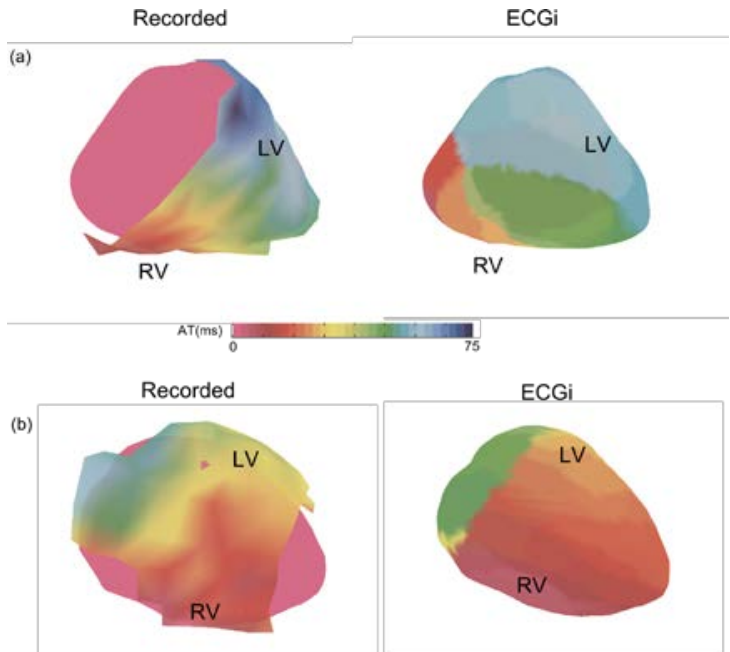

Fig 2. Examples of recorded and ECGi activation maps during (a) complete and (b) incomplete LBBB.

Fig 3 shows the absolute error between recorded and ECGi derived markers across the data. While there was no significant difference in VEU, LVTAT, TAT, or QRSi values ( $p>0.06$ ), RVTAT were 12 to 21 ms longer ( $p$ $<0.001$ ). Indeed, the mean absolute error in VEU was only $5 \pm 8 \mathrm{~ms}$ and in TAT $3 \pm 6 \mathrm{~ms}$.

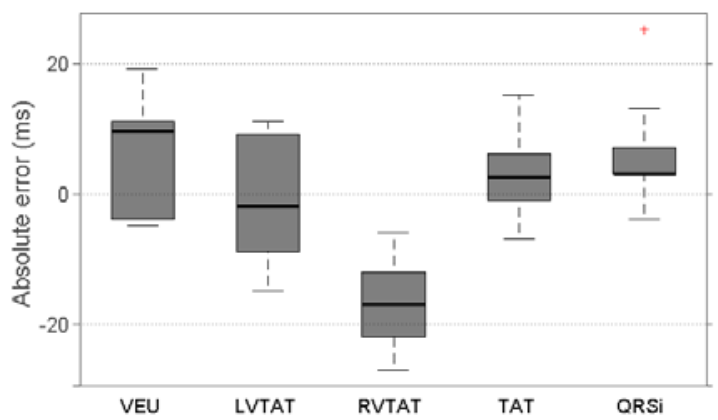

Fig 3. Absolute error between recorded and ECGi derived markers across the spontaneous rhythm signals.

The ability of ECGi to identify incomplete LBBB was evaluated using the same criteria for recorded data (Table 2). All cases of incomplete LBBB were correctly identified, even when delay in LV activation was subtle $\left(\mathrm{VEU}_{\text {recorded }}=17 \mathrm{~ms}\right.$ vs $\mathrm{VEU}_{\mathrm{ECGi}}=10 \mathrm{~ms}$, and $\mathrm{TAT}_{\text {recorded }}$ $=45 \mathrm{~ms}$ vs $\mathrm{TAT}_{\mathrm{ECGi}}=40 \mathrm{~ms}$ ). One complete LBBB case was misidentified as being in incomplete block by ECGi. This case is presented in Fig 4a, and was near the threshold for complete LBBB, both for recorded and ECGi derived markers $\left(\mathrm{VEU}_{\text {recorded }}=46 \mathrm{~ms}\right.$ vs $\mathrm{VEU}_{\mathrm{ECGi}}=36 \mathrm{~ms}$, and $\left.\mathrm{TAT}_{\text {recorded }}=65 \mathrm{~ms} \mathrm{vs} \mathrm{TAT}_{\mathrm{ECGi}}=50 \mathrm{~ms}\right)$.

Table 2 Correct ECGi identification of electrical dyssynchrony

\begin{tabular}{ccc}
\hline Control & $\begin{array}{c}\text { Incomplete } \\
\text { LBBB }\end{array}$ & $\begin{array}{c}\text { Complete } \\
\text { LBBB }\end{array}$ \\
\hline $3 / 3$ & $4 / 4$ & $1 / 2$ \\
\hline
\end{tabular}

\subsection{Resynchronization}

Here the effects of $\mathrm{LV}, \mathrm{RV}$ and $\mathrm{BiV}$ pacing were analyzed for 6 (complete and incomplete) LBBB cases. Table 3 presents a summary of markers derived with example activation maps presented in Fig 2.

Table 3 Summary of experimental recordings post LBBB induction during different pacing protocols.

\begin{tabular}{lccc}
\hline & $\begin{array}{c}\text { LV pacing } \\
(\mathbf{m}=5)\end{array}$ & $\begin{array}{c}\text { RV pacing } \\
(\mathbf{m}=\mathbf{6})\end{array}$ & $\begin{array}{c}\text { BiV pacing } \\
(\mathbf{m}=\mathbf{4})\end{array}$ \\
\hline QRSi (ms) & $99 \pm 14 \dagger$ & $90 \pm 22 \dagger$ & $83 \pm 42 \dagger$ \\
TAT (ms) & $89 \pm 19 \dagger$ & $87 \pm 20 \dagger$ & $76 \pm 39 \dagger$ \\
LVTAT (ms) & $59 \pm 10 \dagger$ & $47 \pm 15 \dagger$ & $54 \pm 25 \dagger$ \\
RVTAT (ms) & $35 \pm 15 \dagger$ & $46 \pm 10 * \dagger$ & $44 \pm 22 * \dagger$ \\
VEU (ms) & $-47 \pm 15^{*} \dagger$ & $36 \pm 19 \dagger$ & $-1 \pm 9 *$ \\
\hline
\end{tabular}

${ }^{*} \mathrm{p}<0.05$ versus complete LBBB. $\dagger \mathrm{p}<0.05$ versus control.

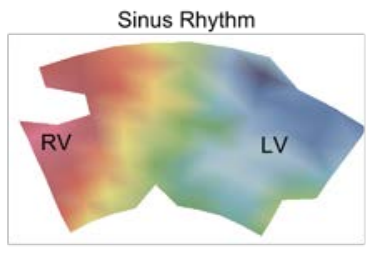

RV pacing

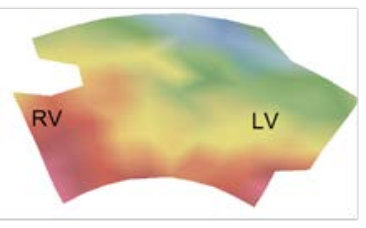

AT (mi)

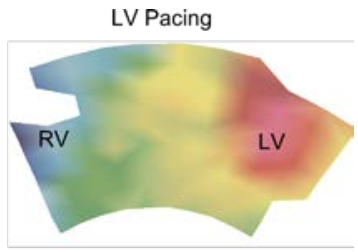

BiV Pacing

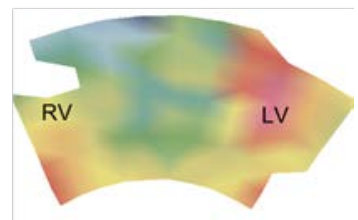

Fig 4. Examples of post LBBB recorded AT maps during sinus rhythm and during different pacing protocols.

As expected, RV pacing produced marker values comparable to complete LBBB in Table 1 (the pattern of activation also similar in Fig 4). LV pacing reversed the activation spread (negative VEU), though there was no improvement to TAT.

$\mathrm{BiV}$ pacing successfully reduced $\mathrm{VEU}$ in all four hearts, compared to the equivalent LBBB model in sinus. Despite this, in two hearts VEU was still $>10 \mathrm{~ms}$, and TAT was not improved. In Fig 2, we see the majority of the epicardium 
is activated under $30 \mathrm{~ms}$ (as in control), however a small area near the base is late to activate, resulting in a large TAT.

\section{Discussion}

In this study, we used a pig model of LBBB with varying levels of electrical dyssynchrony, confirmed by QRS widening, prolongation of activation time and increased LV-RV activation difference. The induced dyssynchrony seen here was similar to a previous porcine and canine models $[7,8]$, and recapitulates abnormalities found in patients with electrical cardiac dyssynchrony. That is, the QRSi produced an average increase of $81 \%$ from the mean baseline QRS width in complete LBBB, and $39 \%$ for incomplete.

By combining this ex-vivo LBBB model with an experimental torso-tank set up, we were able to evaluate the detection of electrical dyssynchrony markers by ECGi. ECGi successfully reconstructed most markers, though the absolute error in some cases was upwards of $20 \mathrm{~ms}$ (Fig 3). This is likely due to one of the known shortcomings of ECGi, in which activation time dispersion is often reduced [9]. While VEU takes an average of the LV and RV, the other markers use the first and last activation times, where ECGi typically fails, to define intervals.

The markers derived with ECGi are also based on a multi-step process. That is reconstructing the epicardial potentials, deriving the activation times from smoothed ECGi potentials, then computing the markers. Error introduced in these steps may be reduced by a) using alternative methods of computing the activation times suited to ECGi, b) directly reconstructing activation times from body surface potentials or c) directly reconstructing the dyssynchrony markers.

Despite the inaccuracy in some of the markers, overall ECGi reliably detected delayed LV activation. Indeed, even the smallest amount of electrical dyssynchrony (QRSi increase of only $10 \%$ ) was captured with ECGi, which would likely go undetected using a standard 12-lead ECG. The patients with these subtle activation changes are typically overlooked for cardiac resynchronization therapy (CRT) but may benefit from this type of procedure, making ECGi a potentially useful tool in this domain.

We also demonstrated here the use of $\mathrm{BiV}$ pacing to restore synchrony to the heart, with pacing leads placed in the typically locations used with CRT. Whilst some success was seen, not all hearts were resynchronized and TAT duration was still longer than ideal. This resynchronization could be improved by optimizing the $\mathrm{LV}$ lead location to the latest site of activation.

Overall, the results presented demonstrate that ECGi can be applied to 1) reliably and noninvasively provide markers for electrical dyssynchrony and 2) optimize, in real-time, the placement and stimulus delay of pacing leads.

\section{Acknowledgements}

This work was supported in part by the French National Research Agency under Contract ANR-10-IAHU04LIRYC. Laura Bear was supported by the Leducq Fondation and grant ANR-10-IAHU-04.

\section{References}

[1] Vernooy K, Cornelussen RN, Verbeek XA, Vanagt WY, van Hunnik A, Kuiper M, et al. Cardiac resynchronization therapy cures dyssynchronopathy in canine left bundlebranch block hearts. Eur Heart J. 2007;28(17):2148-55.

[2] Ploux S, Lumens J, Whinnett Z, Montaudon M, Strom M, Ramanathan C, et al. Noninvasive electrocardiographic mapping to improve patient selection for cardiac resynchronization therapy: beyond QRS duration and left bundle branch block morphology. J Am Coll Cardiol. 2013;61(24):2435-43.

[3] Bakker PF, Meijburg HW, de Vries JW, Mower MM, Thomas AC, Hull ML, Robles De Medina EO, Bredee JJ. (2000) Biventricular pacing in end-stage heart failure improves functional capacity and left ventricular function. J Interv Card Electrophysiol 4:395-404.

[4] Derval N, Steendijk P, Gula LJ, Deplagne A,et al. Optimizing hemodynamics in heart failure patients by systematic screening of left ventricular pacing sites: the lateral left ventricular wall and the coronary sinus are rarely the best sites. Journal of the American College of Cardiology. 2010 Feb 9;55(6):566-75.

[5] Wang Y, Rudy Y. Application of the method of fundamental solutions to potential-based inverse electrocardiography. Ann Biomed Eng. 2006;34(8):1272-88.

[6] Ploux S, Lumens J, Whinnett Z, Montaudon M, Strom M, Ramanathan C, et al. Noninvasive Electrocardiographic Mapping to Improve Patient Selection for Cardiac Resynchronization Therapy. J Am Coll Cardiol. 2013;61(24):2435-43.

[7] Strik M, Van Deursen CJM, Van Middendorp LB, Van Hunnik A, Kuiper M, Auricchio A, et al. Transseptal conduction as an important determinant for cardiac resynchronization therapy, as revealed by extensive electrical mapping in the dyssynchronous canine heart. Circ Arrhythmia Electrophysiol. 2013;6(4):682-9.

[8] Rigol M, Solanes N, Fernandez-Armenta J, Silva E, Doltra A, Duchateau N, et al. Development of a swine model of left bundle branch block for experimental studies of cardiac resynchronization therapy. J Cardiovasc Transl Res. 2013;6:616-22.

[9] Han C, Liu Z, Zhang X, Pogwizd SM, He B. Noninvasive three-dimensional cardiac activation imaging from body surface potential maps: A computational and experimental study on a rabbit model. IEEE Trans Med Im. 2008;27(11):1622-30.

Dr. Laura Bear, IHU-Liryc, Hôpital Xavier Arnozan, Avenue du Haut Lévêque, 33600 Pessac, France.

laura.bear@ihu-liryc.fr 